\title{
Caracterización de viveros de plantas nativas y su posible aporte a la restauración de los talares bonaerenses
}

\author{
Mariela V. Lacoretz ${ }^{1, \bigotimes}$; Cristian Malavert ${ }^{2}$; Carmen Rolandi ${ }^{3}$; Cecilia Zilli ${ }^{1 / 4}$; \\ Nora Madanes ${ }^{1} \&$ Piedad M. Cristiano $^{1}$ \\ ${ }^{1}$ Departamento de Ecología, Genética y Evolución, Facultad de Ciencias Exactas y Naturales, Universidad de Buenos Aires. \\ Buenos Aires, Argentina. ${ }^{2}$ Cátedra de Cultivos Industriales, Facultad de Agronomía, Universidad de Buenos Aires. ${ }^{3}$ Feriado al \\ Planeta. Organización No Gubernamental. Buenos Aires, Argentina. ${ }^{4}$ Naturalista y viverista de plantas nativas.
}

\begin{abstract}
Resumen. Los talares bonaerenses son el principal bosque nativo de la provincia de Buenos Aires; sin embargo, se encuentran en peligro. Dada su extrema vulnerabilidad, no sólo es necesario promover su conservación, sino también su restauración. Los viveros de plantas nativas representan un eslabón clave en los proyectos de restauración. En este trabajo se realizó la primera encuesta a viveros de plantas nativas en el área de distribución de los talares bonaerenses para caracterizarlos y evaluarlos como posibles nodos de restauración. Durante 2019 se realizaron encuestas a estos viveros en forma presencial o en línea. Más de 70\% de los viveros se concentran en el Área Metropolitana de Buenos Aires y tienen entre sus objetivos comercializar, conservar/restaurar o educar. Más de $60 \%$ de los viveros tienen una antigüedad menor a 5 años, un tamaño menor a $500 \mathrm{~m}^{2}$ y no controlan la humedad, la temperatura o la luz de su vivero, pero cuentan con invernaderos. Estas características parecen reflejar un incremento del interés de la sociedad en los últimos años por las plantas nativas que habría provocado el desarrollo de nuevos viveros a escalas pequeñas. Las principales especies del talar son cultivadas en estos viveros, por lo que podrían ser actores clave a la hora de planificar proyectos de restauración de estos bosques. Destacamos la importancia de consolidar una red de colaboración entre viveristas, científicos, áreas protegidas y organismos gubernamentales para restaurar este ecosistema. Es necesario ampliar el alcance de este estudio para conocer los viveros de plantas nativas del resto del país, asociados con otros ecosistemas en peligro y poder evaluar si nuestro país está en condiciones de cumplir sus metas internas y compromisos internacionales de restauración. Esperamos que los resultados de este trabajo promuevan colaboraciones conjuntas para restaurar a los talares bonaerenses y otros ecosistemas de la Argentina.
\end{abstract}

[Palabras clave: bosques nativos, Celtis tala, encuestas, restauración ecológica]

\begin{abstract}
Aвstract. Characterization of native plant nurseries and their possible contribution to the restoration of the talares of Buenos Aires. The talares represent the main native forest of the Buenos Aires province; however, they are in danger. Given their extreme vulnerability, it is not only necessary to promote their conservation, but also their restoration. The native plant nurseries represent a key link in restoration projects. In this work, the first survey of native plant nurseries in the distribution area of talares of Buenos Aires was carried out to characterize and evaluate them as possible restoration nodes. During 2019, surveys to these nurseries were carried out either in person or online. More than $70 \%$ of the nurseries were concentrated in the Buenos Aires Metropolitan Area and their objectives comprise commercialization, conservation/restoration or education. More than $60 \%$ of the nurseries were less than 5 years old and less than $500 \mathrm{~m}^{2}$ in size, and they do not control humidity, temperature and/or light; however, they have greenhouses. These characteristics seem to reflect an increase in society's interest on native plants in recent years, which would have led to the development of new nurseries at small scale. The main plant species of the talares were cultivated in these nurseries, so they could be very important actors when planning restoration projects of these forests. We emphasize the importance of consolidating a network of collaboration between nurseries, scientists, protected areas, and government agencies in order to restore this ecosystem. In this way, it is necessary to expand the scope of this study in order to know the native plant nurseries in the rest of the country, associated with other endangered ecosystems, and to be able to evaluate if Argentina is in a position to meet its internal goals and international restoration commitments. We hope that the results of this work will promote joint collaborations to restore the talares of Buenos Aires and other ecosystems in Argentina.
\end{abstract}

[Keywords: native forests, Celtis tala, surveys, ecological restoration] 


\section{INTRODUCCIÓN}

Los talares bonaerenses, bosques dominados por Celtis tala, son el principal ecosistema boscoso nativo de la Región Pampeana (Matteucci 2012). Estos bosques se extendían desde el norte de la provincia de Buenos Aires, en una estrecha franja costera que llegaba hasta Mar del Plata (Parodi 1940). Sin embargo, al ser la zona más poblada de la Argentina, donde las ciudades crecieron, los talares desaparecieron casi en su totalidad (Lacoretz 2018). Sumado a esto, la extracción de leña y conchilla, la ganadería y la invasión de árboles exóticos hace que hoy los talares se encuentren en peligro de extinción (Mérida and Athor 2006; Guerrero 2019). Estos bosques aumentan la diversidad regional de especies y garantizan servicios ecosistémicos como el secuestro de $\mathrm{CO}_{2}$, la protección de los suelos, la regulación del ciclo hidrológico, brindan sombra para el ganado y espacios de recreación y turismo (Mérida and Athor 2006). Además de promover la conservación de los talares ampliando y creando áreas protegidas y fomentando el ecoturismo y la educación ambiental, es necesario realizar tareas de restauración ecológica en el corto plazo debido al grado de deterioro de este ecosistema boscoso, para preservar su biodiversidad y servicios ambientales (Mérida and Athor 2006).

La restauración ecológica ayuda a restablecer un ecosistema degradado, dañado o destruido (SER 2004). Los proyectos de restauración se basan en el manejo o manipulación de factores bióticos y abióticos a fin de remover amenazas en un ecosistema, facilitar o acelerar la recuperación y aumentar la conectividad con el paisaje (Palmer et al. 2016). En la Argentina, diferentes proyectos de restauración se llevaron a cabo en los últimos años (Rovere 2015; de Paz et al. 2019). En particular, en los talares se desarrollaron proyectos de investigación a pequeña escala en el este (Plaza Behr et al. 2016; Plaza Behr 2017; Azcona 2018; Schrohn 2019) y en el norte de la distribución de los talares (Gasparri et al. 2018). Además, en la Argentina se sancionaron leyes que promueven la restauración de los bosques, como la Ley Nacional de Presupuestos Mínimos de Protección Ambiental de los Bosques Nativos, que creó el Fondo Nacional para el Enriquecimiento y la Conservación de los Bosques Nativos y el Programa Nacional de Protección de los Bosques Nativos (Ley de Bosques, Ley Nro. 26331/2007). También se creó el Plan Nacional de Restauración de Bosques Nativos (PNRBN 2019), que tiene entre sus objetivos alcanzar veinte mil hectáreas anuales bajo restauración a 2030 y elaborar una base de datos de viveros que produzcan plantas nativas. En noviembre de 2020, la provincia de Buenos Aires lanzó el Plan Provincial de Recuperación del Patrimonio Ambiental-Nativas Bonaerenses, que tiene dentro de sus objetivos dejar instalada una capacidad de producción de 300000 plantas por año en la provincia destinados a restauración. Además de sus metas internas, nuestro país está comprometido en distintas iniciativas internacionales relacionadas con la restauración, como el Convenio sobre la Diversidad Biológica (CDB 2010), el Desafío de Bonn (Bonn Challenge 2011) y la Iniciativa 20×20 (Initiative 20x20 2014).

Sin embargo, para que un proyecto de restauración sea exitoso a gran escala y a largo plazo, deben utilizarse estrategias de restauración socialmente robustas, con colaboración de diversos actores sociales (Gross 2006; Ceccon and Pérez 2016; Pérez et al. 2018). La Red de Viveros de Plantas Nativas de Argentina (REVINA) y la Red de Restauración Ecológica de la Argentina (REA) (Pérez et al. 2018) se constituyen como el nexo entre los distintos actores sociales que pueden estar involucrados en la restauración de los ecosistemas de la Argentina. En este contexto, los viveros de plantas nativas podrían representar un actor social clave en los proyectos de restauración de bosques y actuar como nodos de restauración, si entre sus objetivos tienen restaurar el ambiente y producen las plantas nativas necesarias para llevarlo a cabo. Si se piensa en un esquema de árbol, el nodo representaría a los viveros como puntos de origen a partir de los cuales se ramificarían las acciones de restauración. Además, los viveros podrían impartir educación ambiental al promover respuestas a preguntas tales como ¿Por qué cultivar especies nativas? ¿Cuál es el ecosistema característico del lugar donde se ubica el vivero?, y aportar a una construcción social en la cual la restauración forme parte del sentido común (Pérez et al. 2019; Garzón et al. 2020).

Si bien los viveros de plantas nativas están vinculados entre sí a través de la REVINA, no existe hasta la fecha un relevamiento de los mismos en la zona de la distribución de los talares ni en el resto del país. ¿Existen viveros de plantas nativas a lo largo de la distribución original de los talares? ¿Se distribuyen 
homogéneamente? ¿Qué objetivos persiguen? ¿Qué antigüedad y dimensiones poseen? ¿Cultivan las principales especies leñosas nativas del talar? ¿Cuál es su producción anual? En este trabajo se propone: a) caracterizar a los viveros de plantas nativas en el área de distribución de talares y alrededores, y b) a partir de estas características, evaluar si los viveros de plantas nativas pueden ser considerados nodos (o actores importantes) para la restauración, en el área de distribución de los talares bonaerenses.

\section{Materiales y Métodos}

Realizamos una encuesta entre mayo y octubre de 2019 de manera presencial y online a 27 viveros de plantas nativas que se ubican dentro del área de distribución histórica de los talares bonaerenses y cercanías (Figura 1). Alcanzamos nuestra población objetivo: 1) presentando un poster en el 4to Encuentro anual de la REVINA de 2019, en el que se invitaba a los viveristas a contestar la encuesta mediante un código QR, 2) compartiendo el enlace de la encuesta a través de un afiche diseñado a tal efecto en la página de Facebook de la REVINA y en páginas afines (alcance: 13500 personas), 3) enviando mensajes por redes sociales a distintos viveros de plantas nativas del área de estudio, y 4) difundiendo la encuesta en nuestra propia red de investigadores y viveristas. Teniendo en cuenta la última inscripción al encuentro anual del REVINA 2020, estimamos que los viveros de plantas nativas de Buenos Aires son menos de 100 (datos no publicados), por lo que nuestros datos representan alrededor de $30 \%$ de la población objetivo.

Las preguntas fueron cerradas y estuvieron orientadas a indagar sobre las principales características de cada vivero, incluyendo: 1) ubicación, y 2) tipo de vivero (entre ellos, pertenecientesaun comerciodeventaal público o mayorista [comercio], a áreas protegidas, a paisajistas [dedicados a la planificación y el diseño de parques y jardines], a municipios y a organizaciones no gubernamentales [ONG]), 3) objetivo (comercial, educativo, conservación/restauración, social, otros), 4) antigüedad (menos de un año, entre 1 y 5 años, entre 5 y 10 años, más de 10 años), 5) dimensiones (menos de $100 \mathrm{~m}^{2}$, entre 100 y $500 \mathrm{~m}^{2}$, entre 500 y $1000 \mathrm{~m}^{2}$, más de 1000 $\left.\mathrm{m}^{2}\right), 6$ ) si cuenta con invernáculo (si, no), 7) cantidad de personas que trabajan en el vivero (empleados, voluntarios), 8) si poseen control sobre las condiciones ambientales (luz, temperatura, humedad), 9) si cultivan especies de talar (Acacia bonariensis, Acanthosyris spinescens, Celtis tala, Colletia spinosissima, Jodina rhombifolia, Parkinsonia aculeata, Prosopis alba, Sambucus australis, Schinus longifolius, Scutia buxifolia, Zanthoxylum fagara), y 10) producción anual de plantas nativas. Con la información recolectada se calculó la media y el error estándar o porcentaje con respecto al total de viveros de cada variable, según correspondiera. Para las variables antigüedad y tamaño de vivero se calculó su media y error estándar utilizando la marca de clase de cada intervalo. Para las variables tipo de vivero, objetivo y control de condiciones ambientales, en las cuales las categorías no fueron mutuamente excluyentes, se realizaron diagramas de Venn con el paquete venneuler (Wilkinson 2011) con el programa R v.3.6.0 (R Core Team 2019).

\section{Resultados}

El $77 \%$ de los viveros encuestados se concentraron en el Área Metropolitana de Buenos Aires (Figura 1). Por un lado, los principales tipos de viveros de plantas nativas fueron los de tipo comercial (44\%), que se asociaron a proyectos de paisajistas (22\%); por el otro, los vinculados a áreas protegidas (22\%), más relacionados con ONG (19\%) y municipios (7\%) (Figura 2a). Los objetivos de

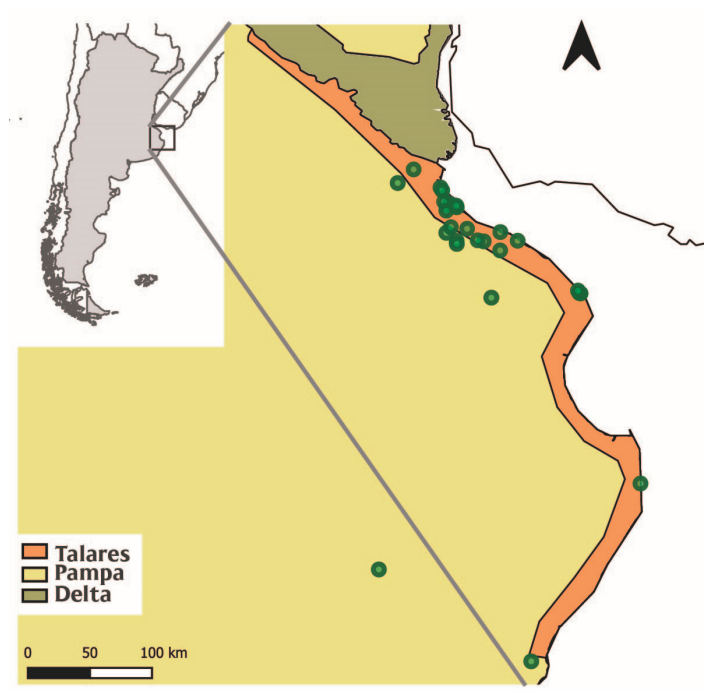

Figura 1. Distribución histórica de los talares en la provincia de Buenos Aires (modificado de Parodi 1940). Los puntos representan los viveros encuestados.

Figure 1. Historical distribution of talares in the province of Buenos Aires (modified from Parodi 1940). The dots represent the plant nurseries surveyed. 
a)

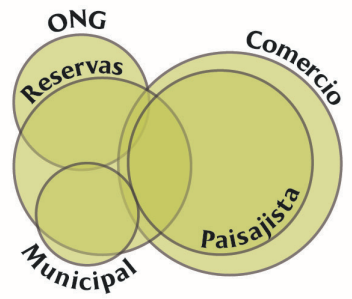

b)

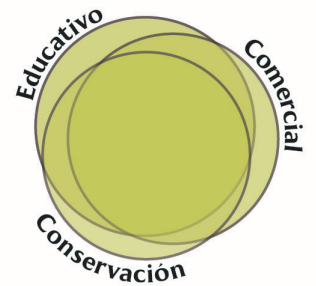

Figura 2. Tipo de vivero (a) y objetivos (b) de los viveros de plantas nativas de la distribución de los talares.

Figure 2. Type of plant nursery (a) and objectives (b) of the native plant nurseries in the talares distribution.

estos viveros fueron múltiples, y estuvieron representados de manera equivalente el objetivo de conservación/restauración $(70 \%)$, el educativo (67\%) y el comercial $(67 \%)$, superponiéndose en un $77 \%$ de los casos al menos dos de los objetivos anteriores (Figura $2 b$ ). Además, $33 \%$ de los viveros tuvieron un objetivo social.

El 66\% de los viveros posee una antigüedad menor a 5 años (antigüedad media: $4.87 \pm 0.81$ años), y casi el $80 \%$ de los mismos tienen un tamaño menor a $500 \mathrm{~m}^{2}$ (tamaño medio: $316 \pm 68$ $\mathrm{m}^{2}$ ) (Figura 3). Las variables antigüedad y tamaño no presentaron una relación aparente entre sí ni con la producción de plantas por vivero (Figura 3). Todos los viveros de mayor antigüedad (más de 10 años) y mayor tamaño (más $1000 \mathrm{~m}^{2}$ ) poseen objetivos de conservación/restauración (Figura 3). En promedio, los viveros tienen 3 empleados, y $39 \%$ cuenta con el apoyo de voluntarios. El $22 \%$ de los viveros no posee control de las variables ambientales y más de la mitad no controla la humedad, la temperatura o la luz de su vivero (Figura 4). No obstante, dentro del vivero, $63 \%$ cuenta con invernaderos de entre 15 y $1500 \mathrm{~m}^{2}$. En la mayoría de los viveros no existe especialización en la producción de plantas nativas, ya que $64 \%$ de los mismos produce tanto herbáceas como arbustos y árboles. La especie leñosa del talar más

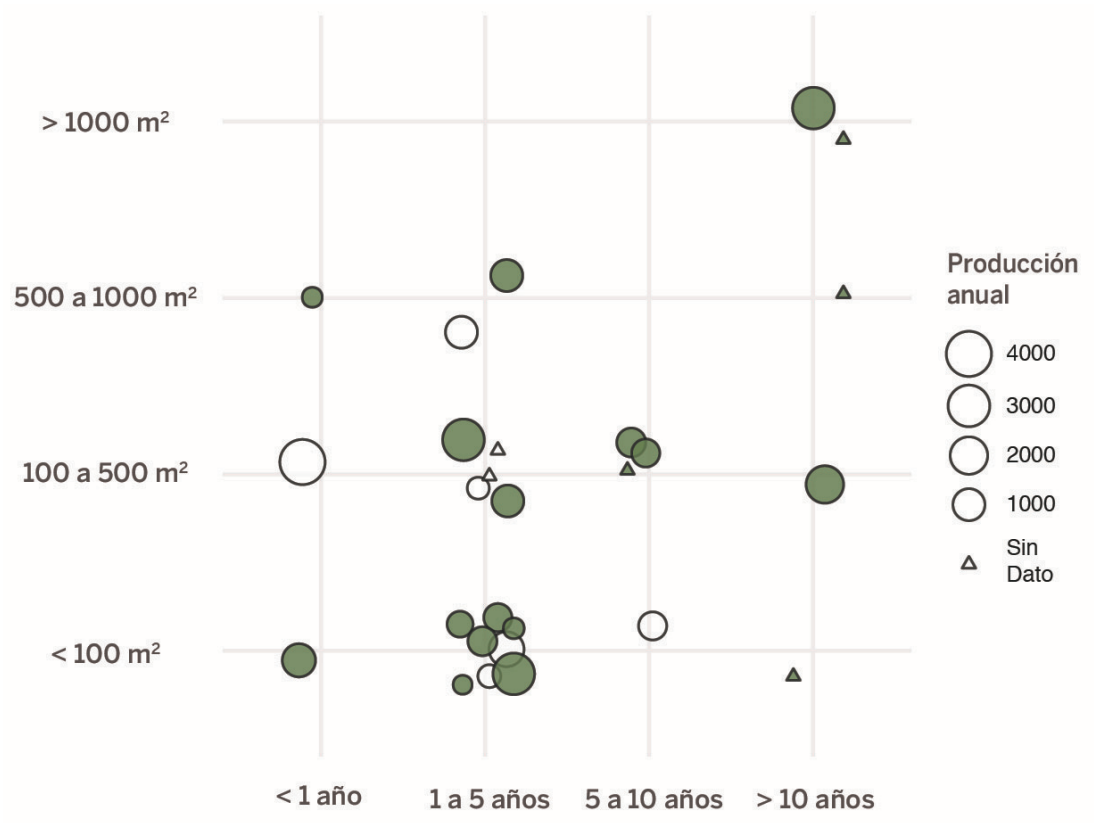

Figura 3. Relación entre el tamaño y la antigüedad de los viveros de plantas nativas de la distribución de los talares. Cada punto representa un vivero, el tamaño de los círculos es proporcional al tamaño de la producción de plantas por vivero y, en caso de no tener el dato de un determinado vivero para esta variable, se indica con un triángulo (sin dato). En color verde se representan los viveros que tienen objetivos de conservación/restauración. Si bien tamaño y antigüedad fueron tomadas en este trabajo como variables categóricas, se agrega un desplazamiento aleatorio de los puntos para mejorar su visualización.

Figure 3. Relationship between the size and age of the native plant nurseries in the talares distribution. Each point represents a nursery, the size of the circles is proportional to the size of the plant production per nursery and, in case we do not have the data of a certain nursery for this variable, it is indicated with a triangle (not available). The green color represents the nurseries that have conservation/restoration objectives. Although size and age were taken in this work as categorical variables, a random displacement of the points is added to improve their visualization. 


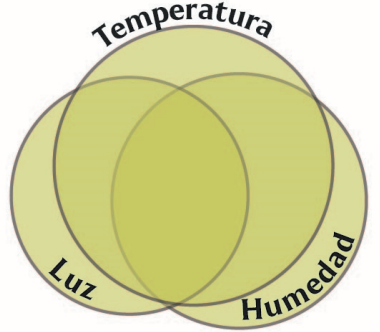

Figura 4. Condiciones ambientales controlados por los viveros de plantas nativas de la distribución de los talares. El 33\% de los viveros controla temperatura, 26\% controla la luz, $30 \%$ controla la humedad, mientras que $22 \%$ no controla ninguna de estas variables. Un $26 \%$ controla al menos 2 variables y $7 \%$ controla las 3 .

Figure 4. Environmental conditions controlled by the native plant nurseries in the talares distribution. Nurseries with temperature control: $33 \%$, light control $26 \%$, humidity control $30 \%, 22 \%$ do not control any of these variables, $26 \%$ control at least 2 variables and $7 \%$ control the three environmental conditions cultivada es Celtis tala, seguida por Schinus longifolius y Acacia bonariensis (Figura 5), llegando a una producción anual promedio de $1139 \pm 258$ plantas nativas por vivero.

\section{Discusión}

En este trabajo se buscó caracterizar a los viveros de plantas nativas en el área de distribución de los talares e identificarlos como posibles nodos de restauración de estos bosques. Los resultados obtenidos muestran que la mayoría de los viveros de plantas nativas relevados se encuentran en el Área Metropolitana de Buenos Aires, son escasos al sur de la provincia y no existen registros en el norte. Los mismos se dividen en los que se consideran comerciales, asociados con los paisajistas, y aquellos de áreas protegidas,
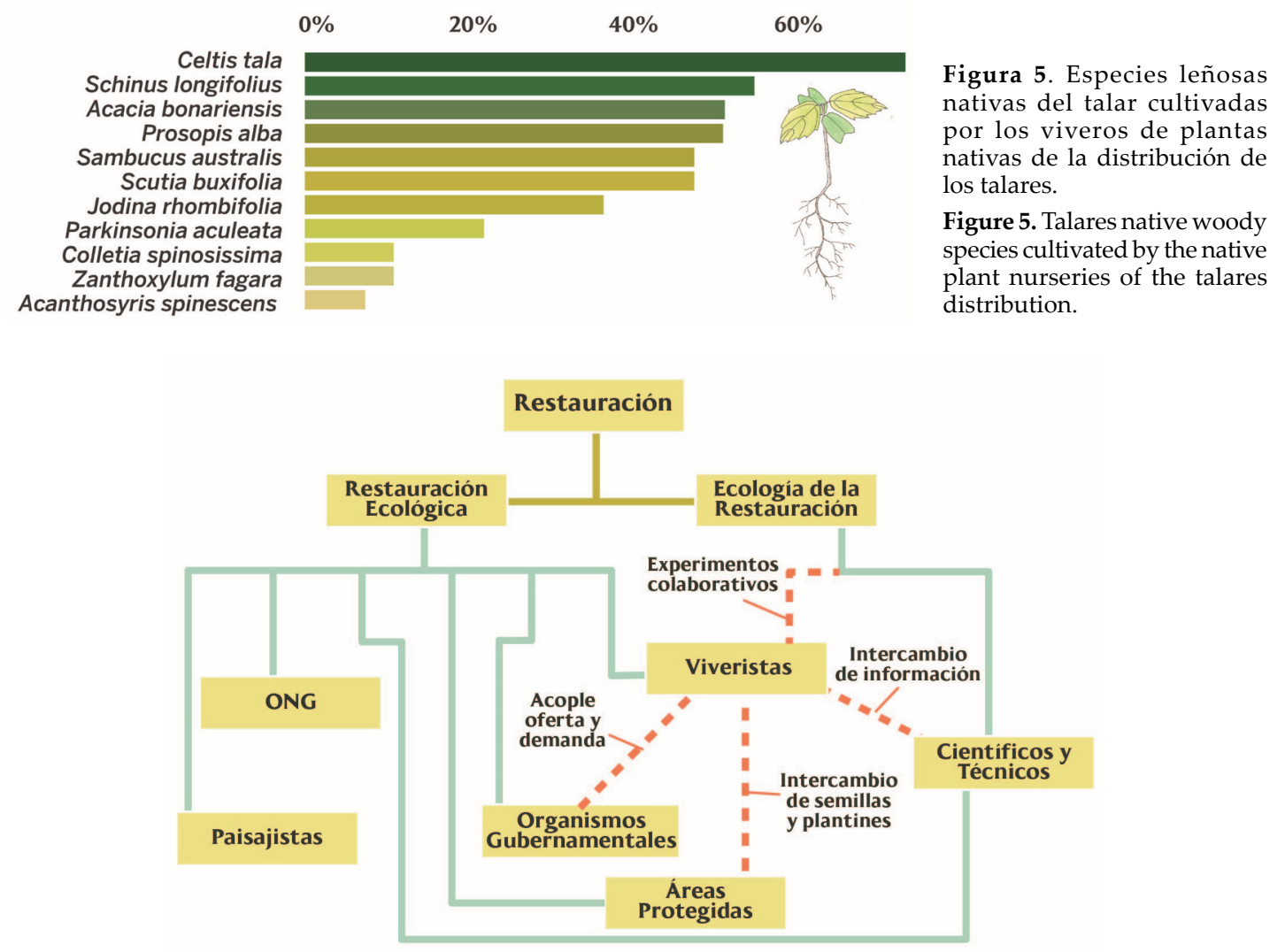

Figura 6. Esquema conceptual de los principales actores involucrados en el proceso de restauración de los talares bonaerenses, con posibilidad de extrapolarlo a otros ecosistemas boscosos. Las líneas verdes conectan a los dos componentes de la restauración, la restauración ecológica (práctica) y la ecología de la restauración (teoría ecológica). Las líneas celestes representan cómo los diversos actores aportan a uno o ambos componentes de la restauración. Las líneas punteadas resaltan aquellas interacciones a reforzar dentro de esta red con respecto a los viveristas de plantas nativas.

Figure 6. Conceptual scheme of the main actors involved in the restoration process of talares of Buenos Aires, with the possibility of extrapolation to other forest ecosystems. The green lines connect the two components of restoration ecology (practice) and ecological restoration (theory). The light blue lines represent how the various actors contribute to one or both components of restoration. Dotted lines highlight those interactions to be reinforced within this network with respect to native plant nurseries. 
asociados con municipios y ONG. Se observó que poseen múltiples objetivos compartidos y que la gran mayoría tiene como meta conservar y restaurar el ambiente. En general, tienen poco personal y antigüedad, sus dimensiones son pequeñas, no especializan su producción y controlan de manera limitada los factores ambientales. Estas características parecen reflejar un incremento del interés de la sociedad en los últimos años por las plantas nativas, que habría provocado el desarrollo de nuevos viveros a escalas pequeñas. Este tipo de encuestas se ha realizado en otros países de América, tales como México (Benítez et al. 2002), Chile (CONAF), Brasil (Vidal et al. 2020) y Estados Unidos (Brzuszek and Harkess 2009), pero no existen datos disponibles en la distribución de los talares ni en el resto de la Argentina.

Las principales especies leñosas del talar son cultivadas en estos viveros, por lo que podrían ser actores muy importantes a la hora de planificar proyectos de restauración de los talares bonaerenses. La especie dominante del talar, Celtis tala, es cultivada por más del $60 \%$ de los viveros de plantas nativas encuestados. Además, gran parte de los viveros también cultivan Schinus longifolius, Acacia bonariensis y Proposis alba, y en menor proporción Sambucus australis, Scutia buxifolia, y Jodina rhombifolia. Esta última especie fue mencionada como de muy difícil propagación en vivero mediante semilla (comunicación personal de viveristas), aunque constituye una de las especies con mayor abundancia de los talares (Lacoretz 2018).

La producción anual promedio en estos viveros es pequeña si se la compara, por ejemplo, con Brasil, donde $52 \%$ de los viveros de plantas nativas produce $50000 \mathrm{o}$ más plantas por año (Moreira da Silva et al. 2017). Si se considera que la media obtenida por vivero en este trabajo fue de 1139 plantas nativas de distintas formas de vida (árboles, arbustos y herbáceas), los 27 viveros evaluados en conjunto producirían cerca de 30000 plantas por año. Si se tiene en cuenta, además, que un talar maduro y conservado en la localidad de Magdalena tiene un promedio de 760 árboles por hectárea (datos aún no publicados), la producción actual de los viveros evaluados alcanzaría idealmente a restaurar 40 ha de talar por año. Sin embargo, el número de plantines debería ser más grande aun para alcanzar esa densidad de árboles. Esto se debe, por un lado, a que la densidad estimada (760 por hectárea) es de árboles y que la producción calculada anual de los viveros no diferenció entre formas de vida. Por otro lado, la producción debería ser mayor ya que, a pesar de que existe un escaso conocimiento de supervivencia de plantas de especies nativas en proyectos de restauración de los talares, se estima que es bajo. Por ejemplo, se vio que la supervivencia media de Celtis tala fue de $70 \%$ en los primeros 3 años en claros de talares invadidos por ligustro (Plaza Behr et al. 2016) y sólo de 15\% en canteras (Azcona 2018), por lo que se debería tener en cuenta para calcular la cantidad de plantines necesarios. Asimismo, como se dijo antes, el Plan Provincial de Recuperación del Patrimonio AmbientalNativas Bonaerenses tiene como uno de sus objetivos producir 300000 plantas por año en la provincia, por lo cual la capacidad de los viveros evaluados sería un $10 \%$ de lo que pretende alcanzar este plan. Por lo tanto, la producción anual de plantas debería aumentar si se desean realizar acciones de restauración de los talares a gran escala y cumplir con los objetivos de restauración propuestos por la provincia.

Teniendo en cuenta el grado de perturbación que han sufrido los talares bonaerenses (Mérida and Athor 2006; Guerrero 2019), consideramos que es necesario promover de manera activa los proyectos de restauración en la zona de distribución original de este bosque. Los proyectos de restauración de los talares necesitan de los productores de plantas nativas. El involucramiento de los viveristas es factible dado que la mayoría tiene como finalidad la conservación y la restauración, independientemente de si son viveros comerciales o no. Además, poseen experiencia en el cultivo de las plantas nativas de los talares, aunque esta información no se encuentre fácilmente disponible. De esta manera, los viveros de plantas nativas podrían ser considerados nodos (o actores importantes) para la restauración de los talares bonaerenses, ya que tienen el objetivo de restaurar el ambiente $y$, además, producen las plantas propias del talar.

A pesar de que existe esta oportunidad, hay ciertas limitantes para que los viveros de plantas nativas constituyan una red de nodos de restauración. Por un lado, estos viveros se ubican principalmente en el Área Metropolitana de Buenos Aires, por lo que se debería promover la creación de más viveros de plantas nativas e incentivar la conversión de viveros tradicionales hacia la producción de especies nativas en el resto de 
la distribución de los talares. Sin embargo, no existe un relevamiento preciso y disponible de la superficie total que ocupan los talares en la actualidad ni una estimación de cuál fue su cobertura histórica. Esta información es necesaria para saber dónde se encuentran las áreas en las cuales sería posible realizar acciones de restauración. Por otro lado, también es necesario fomentar que los viveros de plantas nativas aumenten su capacidad de producción y especialización, como está siendo sugerido en otras partes del mundo (Friday et al. 2015; Haase and Davis 2017; Norton et al. 2018). Por último, actualmente no existe una lista sistematizada y publicada de proyectos de restauración en el área ocupada históricamente por los talares ni información sobre el grado de involucramiento de los viveros en estos proyectos.

Los viveristas son uno de los actores sociales que pueden participar de la restauración de un ambiente (Figura 6). Los diferentes actores sociales se involucran en la restauración a través de su práctica, restauración ecológica, o el desarrollo de su teoría, ecología de la restauración, nutriéndose mutuamente, aunque no siempre bien conectados (SER 2004; Palmer et al. 2016; Miller et al. 2017; Cooke et al. 2019; Figura 6). En este marco conceptual, destacamos la importancia que tienen los viveristas en la red de actores. Por un lado, los viveristas producen las plantas nativas que se necesitan para restaurar un ambiente activamente (Haase and Davis 2017); por otro lado, proponemos que ciertas interacciones de los viveristas en esta red se refuercen (Figura 6, líneas punteadas). Por ejemplo, los viveristas desarrollaron técnicas de conservación y germinación de semillas, establecimiento de plántulas y, además, conocen qué vacíos de información existen. Este conocimiento no se encuentra documentado y sería conveniente la interacción con grupos de científicos y técnicos que lo recopilen e investiguen (Figura 6, intercambio de información). En este sentido, el conocimiento sobre la ecofisiología de las semillas y el establecimiento de las plántulas permitirá estandarizar la producción de especies nativas en vivero y desarrollar proyectos de restauración in situ (Khurana and Singh 2001; Kildisheva et al. 2020). Los viveristas también podrían contribuir a la teoría de la restauración ecológica si participaran de experimentos colaborativos en coordinación con grupos de investigación (Figura 6, experimentos colaborativos). Además, al vincularse con áreas protegidas, podrían asegurarse la provisión de semillas de alta calidad, que suele ser una gran limitante en la producción de nativas (LeónLobos et al. 2020) y, a su vez, las reservas podrían proveerse de los plantines de estos viveros (Figura 6, intercambio de semillas y plantines). Con ciertas excepciones, en restauración se recomienda el uso de semillas de procedencia local para evitar mala adaptación y contaminación genética (Pastorino et al. 2017). Por esto, es conveniente que los viveros utilicen semillas de sitios de referencia cercanos al sitio a restaurar (McKay et al. 2005), para lo cual es necesario generar normativas y convenios con áreas protegidas próximas a los viveros que faciliten la disponibilidad de las semillas. Por último, es imprescindible elaborar planes de restauración definidos a largo plazo (Bannister et al. 2018), que sean fomentados desde los organismos gubernamentales, ya que permitiría acoplar la oferta con la demanda de plantas nativas (Handel 2017). De esta manera, los viveristas serían capaces de planificar y aumentar su producción (Figura 6, acople oferta y demanda).

La activa participación de los organismos gubernamentales es fundamental para llevar adelante un proyecto de restauración (Aronson et al. 2011). En este sentido, la Argentina ha hecho grandes avances sancionando la Ley de Bosques (Ley de Bosques, Ley Nro. 26331/2007), creando el Plan Nacional de Restauración de Bosques Nativos (PNRBN 2019) y comprometiéndose a diversas iniciativas internacionales (CDB 2010; Bonn Challenge 2011; Initiative 20x20 2014). Sin embargo, la Argentina declara que mediante la Ley de Bosques financiará los compromisos asumidos en la Iniciativa 20×20, aunque sólo se asignó 3\% de su presupuesto a su aplicación en 2020 (FARN 2019; Ley de Presupuesto General de Argentina 2019). ¿Podrá la Argentina cumplir con los compromisos asumidos? ¿Cuál es la magnitud de la producción de plantas nativas actualmente en el país? La experiencia de Chile y Brasil, donde hace años se realizan censos de la producción de viveros de nativas, revela que a los países vecinos les queda un gran recorrido por hacer para poder cumplir con sus compromisos de restauración internacionales (Moreira da Silva et al. 2017; Bannister et al. 2018; Acevedo et al. 2021). Por esto, dado que en la Argentina desconocemos cuántos viveros de nativas existen, qué especies nativas y cuántas plantas se producen anualmente para hacer frente a los 
distintos desafíos, es importante que se realice un relevamiento de viveros de plantas nativas a nivel nacional. Además, es fundamental que se respeten los presupuestos asignados por ley para garantizar el cumplimiento de las distintas metas. Por último, también se debe fomentar la colaboración entre diversos actores sociales y así tener la posibilidad de restaurar los talares bonaerenses y otros ecosistemas amenazados de la Argentina.

Agradecimientos. Estamos muy agradecidos con los revisores anónimos por su interés en nuestro trabajo y por los valiosos comentarios que lo enriquecieron en gran medida. Agradecemos también a todos los viveristas que se tomaron el tiempo para completar la encuesta. A la Red de Viveros de Plantas Nativas de Argentina (REVINA), al Grupo de Árboles Nativos de Aves Argentinas y a todos aquellos que nos ayudaron a difundir la encuesta a través de sus redes de contactos. Por último, agradecemos a I. Villanova, V. Diaz Villa y R. Burkart por sus comentarios y sugerencias.

\section{REFERENCIAS}

Acevedo, M., C. Álvarez-Maldini, R. K. Dumroese, J. R. Bannister, E. Cartes, and M. González. 2021. Native Plant Production in Chile. Is It Possible to Achieve Restoration Goals by 2035? Land 10:71. https://doi.org/10.3390/ land10010071.

Aronson, J., P. H. S. Brancalion, G. Durigan, R. R. Rodrigues, V. L. Engel, M. Tabarelli, J. M. D. Torezan, S. Gandolfi, A. C. G. de Melo, P. Y. Kageyama, M. C. M. Marques, A. G. Nave, S. V. Martins, F. B. Gandara, A. Reis, L. M. Barbosa, and F. R. Scarano. 2011. What Role Should Government Regulation Play in Ecological Restoration? Ongoing Debate in São Paulo State, Brazil. Restoration Ecology 19:690-695. https://doi.org/10.1111//.1526-100X.2011.00815.x

Azcona, M. 2018. Evaluación de técnicas de rehabilitación de áreas degradadas en los talares de Magdalena y Punta Indio. Trabajo final de grado. Facultad de Ciencias Agrarias y Forestales. UNLP. Pp. 39

Bannister, J. R., R. Vargas-Gaete, J. F. Ovalle, M. Acevedo, A. Fuentes-Ramirez, P. J. Donoso, A. Promis, and C. SmithRamírez. 2018. Major bottlenecks for the restoration of natural forests in Chile. Restoration Ecology 26:1039-1044. https://doi.org/10.1111/rec.12880.

Benítez, G., M. Equihua, and M. T. Pulido-Salas. 2002. Diagnóstico de la situación de los viveros oficiales de Veracruz y su papel para apoyar programas de reforestación y restauración. Revista Chapingo Serie Ciencias Forestales y del Ambiente 8:5-12.

Bonn Challenge. 2011. Bonn Challenge. Restore our future. URL: tinyurl.com/3prm4mw3.

Brzuszek, R. F., and R. L. Harkess. 2009. Green industry survey of native plant marketing in the southeastern United States. HortTechnology 19:168-172. https://doi.org/10.21273/hortsci.19.1.168.

CDB. 2010. Convention on Biological Diversity. URL: cbd.int/countries/profile/?country=ar.

Ceccon, E., and D. R. Pérez. 2016. Más allá de la ecología de la restauración: perspectivas sociales en América Latina y el Caribe Sociedad Iberoamericana y del Caribe de Restauración Ecológica. First edition. Vázquez Mazzini Editores, Buenos Aires, Argentina.

CONAF. Corporación Nacional Forestal. URL: tinyurl.com/ysfsvtd9.

Cooke, S. J., J. R. Bennett, and H. P. Jones. 2019. We have a long way to go if we want to realize the promise of the "Decade on Ecosystem Restoration". Conservation Science and Practice 1:1-5. https://doi.org/10.1111/csp2.129.

de Paz, M., M. Gobbi, and E. Raffaele. 2019. Revisión de las experiencias de revegetación con fines de restauración en bosques de la Argentina. Ecología Austral 29:194-207. https://doi.org/10.25260/ea.19.29.2.0.689.

FARN. 2019. Fundación Ambiente y Recursos Naturales. La Ley de Bosques, cada vez más desfinanciada. URL: tinyurl.com $/ 5 \mathrm{k} 4$ tpthp.

Friday, J. B., S. Cordell, C. P. Giardina, F. Inman-Narahari, N. Koch, J. J. K. Leary, C. M. Litton, and C. Trauernicht. 2015. Future directions for forest restoration in Hawai'i. New Forests 46:733-746. https://doi.org/10.1007/s11056-0159507-3.

Garzón, N. V., C. H. Rodríguez León, E. Ceccon, and D. R. Pérez. 2020. Ecological restoration-based education in the Colombian Amazon: Toward a new society-nature relationship. Restoration Ecology. https://doi.org/10.1111/ rec.13216.

Gasparri, B., G. R. Tourón, M. Fugardo, M. E. Ghelfi, S. Etulain, A. Faccioli, L. Cristaldo, D. Del Río, M. Vitale, G. Bryant, F. Adrio, and C. Rostagno. 2018. Restauración del talar de barranca. El caso del Parque Natural Municipal Barranca de la Quinta Los Ombúes, San Isidro, Buenos Aires, Argentina. Historia Natural (tercera serie) 8:65-88.

Gross, M. 2006. Beyond expertise: Ecological science and the making of socially robust restoration strategies. Journal for Nature Conservation 14:172-179. https://doi.org/10.1016/j.jnc.2006.05.004.

Guerrero, E. L. 2019. The talares of Zárate (Buenos Aires Province, Argentina). A history of losses and a committed future. Revista del Museo Argentino de Ciencias Naturales, Nueva Serie 21:29-44. https://doi.org/10.22179/ REVMACN.21.616.

Haase, D. L., and A. S. Davis. 2017. Developing and supporting quality nursery facilities and staff are necessary to meet global forest and landscape restoration needs. Reforesta 4:69-93. https://doi.org/10.21750/ REFOR.4.06.45.

Handel, S. N. 2017. Growing pains: Hopes and heartaches at native plant nurseries. Ecological Restoration 35:279-280. https://doi.org/10.3368/er.35.4.279.

Initiative 20x20. 2014. URL: initiative20x20.org/regions-countries/argentina. 
Khurana, E., and J. S. Singh. 2001. Ecology of seed and seedling growth for conservation and restoration of tropical dry forest: A review. Environmental Conservation 28:39-52. https://doi.org/10.1017/S0376892901000042.

Kildisheva, O. A., K. W. Dixon, F. A. O. Silveira, T. Chapman, A. Di Sacco, A. Mondoni, S. R. Turner, and A. T. Cross. 2020. Dormancy and germination: making every seed count in restoration. Restoration Ecology 28:S256-S265. https: //doi.org/10.1111/rec.13140.

Lacoretz, M. V. 2018. Análisis de la diversidad de la comunidad de aves de los montes de tala del este de la provincia de Buenos Aires y su relación con la estructura del hábitat. Tesis doctoral. Universidad de Buenos Aires, Argentina. Pp. 148.

León-Lobos, P., M. A. Bustamante-Sánchez, C. R. Nelson, D. Alarcón, R. Hasbún, M. Way, H. W. Pritchard, and J. J. Armesto. 2020. Lack of adequate seed supply is a major bottleneck for effective ecosystem restoration in Chile: friendly amendment to Bannister et al. (2018). Restoration Ecology 28:277-281. https://doi.org/10.1111/rec.13113.

Ley de Presupuesto General de Argentina. 2019. URL: minhacienda.gob.ar/onp/presupuestos/2020.

Ley Nro. 26331. 2007. Presupuestos mínimos de protección ambiental de los bosques nativos. URL: tinyurl.com/ mdakudjy.

Matteucci, S. D. 2012. Ecorregión Pampa. Pp. 391-445 in J. Morello, S. Matteucci, A. Rodríguez and M. Silva (eds.). Ecorregiones y complejos ecosistémicos argentinos. Orientación Gráfica Editora, Buenos Aires, Argentina.

McKay, J. K., C. E. Christian, S. Harrison, and K. J. Rice. 2005. 'How local is local?' - A review of practical and conceptual issues in the genetics of restoration. Restoration Ecology 13:432-440. https://doi.org/10.1111/j.1526100X.2005.00058.x.

Mérida, E., and J. Athor (eds.). 2006. Talares bonaerenses y su conservación. First edition. Fundación de Historia Natural Félix de Azara, Buenos Aires, Argentina.

Miller, B. P., E. A. Sinclair, M. H. M. Menz, C. P. Elliott, E. Bunn, L. E. Commander, E. Dalziell, E. David, B. Davis, T. E. Erickson, P. J. Golos, S. L. Krauss, W. Lewandrowski, C. E. Mayence, L. Merino-Martín, D. J. Merritt, P. G. Nevill, R. D. Phillips, A. L. Ritchie, S. Ruoss, and J. C. Stevens. 2017. A framework for the practical science necessary to restore sustainable, resilient, and biodiverse ecosystems. Restoration Ecology 25:605-617. https://doi.org/10.1111/rec.1247.

Moreira da Silva, A. P., D. Schweizer, H. Rodrigues Marques, A. M. Cordeiro Teixeira, T. V. M. Nascente dos Santos, R. H. R. Sambuichi, C. G. Badari, U. Gaudare, and P. H. S. Brancalion. 2017. Can current native tree seedling production and infrastructure meet an increasing forest restoration demand in Brazil? Restoration Ecology 25:509-515. https: //doi.org/10.1111/rec.12470.

Norton, B. D. A., J. Butt, and D. O. Bergin. 2018. Upscaling restoration of native biodiversity: A New Zealand perspective. Ecological Management and Restoration 19:26-35. https://doi.org/10.1111/emr.12316.

Palmer, M. A., J. B. Zedler, and D. A. Falk. 2016. Foundations of restoration ecology. Second edition. Island Press, Washington, USA. https://doi.org/10.5822/978-1-61091-698-1.

Parodi, L. 1940. Distribución geográfica de los talares de la Provincia de Buenos Aires. Darwiniana 4:33-56.

Pastorino, M., A. Aparicio, M. Azpilicueta, C. Soliani, and P. Marchelli. 2017. Genética de la restauración: tendiendo puentes entre la; investigación y la gestión. Pp. 147-152 in G. Zuleta, A. Rovere and F. Mollard (eds.). SIACRE-2015 Aportes y Conclusiones: Tomando decisiones para revertir la degradación ambiental. Vázquez Mazzini Editores, Buenos Aires, Argentina.

Pérez, D., P. Meli, D. Renison, F. R. Barri, A. M. Beider, G. A. Burgueño, A. D. D. Galván, S. Dardelli, M. de Paz, F. M. Farinaccio, G. Papazian, M. Sirombra, and R. Torres. 2018. La Red de Restauración Ecológica de la Argentina (REA): Avances, vacíos y rumbo a seguir. Ecología Austral 28:353-360. https://doi.org/10.25260/EA.18.28.2.0.659.

Pérez, D. R., F. Del Mar González, M. E. R. Araujo, D. A. Paredes, and E. Meinardi. 2019. Restoration of society-nature relationship based on education:A model and progress in Patagonian Drylands. Ecological Restoration 37:182-191. https://doi.org/10.3368/er.37.3.182

Plaza Behr, M. C. 2017. Evaluación de estrategias de rehabilitación de los bosques de Celtis ehrenbergiana "talares" en canteras de conchilla abandonadas en el partido de Castelli, Buenos Aires. Trabajo final de grado. Facultad de Ciencias Agrarias y Forestales. UNLP. Pp. 36.

Plaza Behr, M. C., C. A. Pérez, J. F. Goya, M. Azcona and M. F. Arturi. 2016. Plantación de Celtis ehrenbergiana como técnica de recuperación de bosques invadidos por Ligustrum lucidum en los talares del NE de Buenos Aires. Ecología Austral 26:171-177. https://doi.org/10.25260/EA.16.26.2.0.176.

PNRBN. 2019. Plan Nacional de Restauración de Bosques Nativos. Resolución 267 / 2019. Secretaria de Gobierno de Ambiente y Desarrollo Sustentable, Argentina. URL: tinyurl.com/2cecsjn4.

R Core Team. 2019. R: A language and environment for statistical computing. R v.3.6.0. R Foundation for Statistical Computing, Vienna, Austria. URL: R-project.org.

Rovere, A. E. 2015. Review of the science and practice of restoration in Argentina: Increasing awareness of the discipline. Restoration Ecology 23:508-512. https://doi.org/10.1111/rec.12240.

SER. 2004. Principios de SER international sobre la restauración ecológica. Society for Ecological Restoration International.

Schrohn, H. C. 2019. Plantaciones de especies nativas para la rehabilitación de canteras de conchilla en Castelli, provincia de Buenos Aires. Trabajo final de grado. Facultad de Ciencias Agrarias y Forestales. UNLP. Pp. 50.

Vidal, C. Y., R. P. Naves, R. A. G. Viani, and R. R. Rodrigues. 2020. Assessment of the nursery species pool for restoring landscapes in southeastern Brazil. Restoration Ecology 28:427-434. https://doi.org/10.1111/rec.13096.

Wilkinson. 2011. venneuler: Venn and Euler Diagrams. R package version 1.1-0. URL: CRAN.R-project.org/ package $=$ venneuler. 